Carla Regina de S. Teixeira, Maria Lúcia Zanetti,

Camila de Moraes, Plínio Tadeu Istilli,

Gabriel Guidorizzi Zanetti, Tânia Alves C. Becker e

Jennifer Vieira P. Marques

\title{
PERCEPÇÃO DO AMBIENTE PARA A PRÁTICA DE ATIVIDADE FÍSICA DE PESSOAS COM DIABETES MELLITUS
}

Recebido em: 14/08/2017

Aceito em: 10/05/2018

\author{
Paula Parisi Hodniki ${ }^{1}$ \\ Carla Regina de Souza Teixeira ${ }^{2}$ \\ Maria Lúcia Zanetti ${ }^{3}$ \\ Camila de Moraes ${ }^{4}$ \\ Plínio Tadeu Istilli ${ }^{5}$ \\ Gabriel Guidorizzi Zanetti ${ }^{6}$ \\ Tânia Alves Canata Becker ${ }^{7}$ \\ Jennifer Vieira Paschoalin Marques ${ }^{8}$ \\ Universidade de São Paulo (USP) \\ Ribeirão Preto - SP - Brasil
}

RESUMO: Este estudo analisou a percepção do ambiente para a prática de atividade física de pessoas com diabetes mellitus tipo 2 de uma cidade do interior paulista. Foram entrevistadas 86 pessoas, quanto aos dados sociodemográficos, Questionário Internacional de Atividade Física, e a Escala de Percepção do Ambiente adaptada. A maioria das pessoas estão acima dos 60 anos de idade, são mulheres, aposentados, baixa renda e não atingiram 150 minutos de prática de atividade física por semana. As pessoas que percebem acesso a conveniências mais próximas à sua residência são mais ativas no tempo de lazer do que as que não percebem. Assim como, as que recebem convite de vizinhos e/ou amigos têm mais chance de praticarem atividade física do que as pessoas que não recebem. As pessoas que percebem acesso a conveniências longe de sua residência e ruas planas próximas à sua residência têm mais chance de praticar caminhada como forma de deslocamento do que as pessoas que não percebem. Há necessidade de adequação dos espaços públicos que favoreça a motivação, o ambiente físico e social para a prática de atividade física de pessoas com diabetes mellitus.

PALAVRAS CHAVE: Exercício. Diabetes Mellitus. Meio Ambiente.

\footnotetext{
${ }^{1}$ Mestre em Ciências, Membro do Grupo de Pesquisa em Enfermagem e Diabetes Mellitus.

${ }^{2} \mathrm{PhD}$ em Enfermagem, Membro do Grupo de Pesquisa em Enfermagem e Diabetes Mellitus.

${ }^{3} \mathrm{PhD}$ em Enfermagem, Membro do Grupo de Pesquisa em Enfermagem e Diabetes Mellitus.

${ }^{4}$ Doutora em Ciências da Motricidade, Membro do Grupo de Estudo e Pesquisa em Exercício Físico para

Condições Especiais de Saúde.

${ }^{5}$ Mestre em Ciências, Membro do Grupo de Pesquisa em Enfermagem e Diabetes Mellitus.

${ }^{6}$ Membro do Grupo de Pesquisa em Enfermagem e Diabetes Mellitus.

${ }^{7}$ Doutora em Ciências, membro do Grupo de Pesquisa em Enfermagem e Diabetes Mellitus.

${ }^{8}$ Membro do Grupo de Pesquisa em Enfermagem e Diabetes Mellitus.
} 
Carla Regina de S. Teixeira, Maria Lúcia Zanetti,

Camila de Moraes, Plínio Tadeu Istilli,

Gabriel Guidorizzi Zanetti, Tânia Alves C. Becker e

Jennifer Vieira P. Marques

\section{PERCEPTION OF THE ENVIRONMENT FOR PHYSICAL ACTIVITY OF PEOPLE WITH DIABETES MELLITUS}

ABSTRACT: This study analyzed the perception of the environment for the practice of physical activity of people with type 2 diabetes mellitus of a city in the interior of São Paulo. A total of 86 people were interviewed regarding sociodemographic data, the International Physical Activity Questionnaire, and the adapted Environmental Perception Scale. Most people are over 60 years of age, are women, retirees, low income and have not reached 150 minutes of physical activity per week. People who perceive access to conveniences closer to their home are more active at leisure than those they do not realize. As well, those who receive invitations from neighbors and / or friends are more likely to engage in physical activity than people who do not. People who experience access to conveniences far from their home and flat streets near their home are more likely to practice walking as a form of travel than people they do not notice. There is a need for adaptation of the public spaces that favors the motivation, the physical and social environment for the practice of physical activity of people with diabetes mellitus.

KEYWORDS: Exercise. Diabetes Mellitus. Environment.

\section{Introdução}

A inatividade física está entre os principais fatores de riscos comportamentais modificáveis para as doenças crônicas não transmissíveis DCNT (MALTA et al., 2015). Destaca-se o diabetes mellitus (DM), que está entre as quatro principais DCNT, somada as doenças do aparelho circulatório, o câncer e as doenças respiratórias crônicas (WHO, 2014). Nessa direção, a prática de atividade física regular é uma importante estratégia do tratamento não farmacológico do DM, pois promove a melhora da sensibilidade dos tecidos à insulina, facilita o metabolismo glicídico, melhora o nível de colesterol e a qualidade de vida, podendo contribuir para a redução dos riscos cardiovasculares (COLBERG; HERNANDEZ; SHAHZAD, 2013).

Embora evidências reforcem o benefício da prática regular de atividade física, a sua promoção é dificultada devido à necessidade de planejamento baseado em evidências para intervenção e implementação de políticas necessárias, como criação e 
Carla Regina de S. Teixeira, Maria Lúcia Zanetti,

Camila de Moraes, Plínio Tadeu Istilli,

Gabriel Guidorizzi Zanetti, Tânia Alves C. Becker e

Jennifer Vieira P. Marques

melhoria de acesso aos locais de atividade física, com atividades informativas de sensibilização e incentivo às práticas de transporte ativo, associando diversas abordagens que podem incrementar o nível de atividade física entre as pessoas de várias idades e de diferentes grupos sociais, especialmente entre países de baixa renda (SALLIS et al., 2016).

Porém, mais estudos são necessários para fornecer um entendimento completo das relações físicas e sociais entre o ambiente e o estilo de vida para auxiliar profissionais a focarem as intervenções com intuito do aumento do uso do ambiente para prática de atividade física em grupos específicos da população (FERMINO; REIS, 2014). Estes estudos por sua vez, devem proporcionar o entrosamento entre a pesquisa e o âmbito da prática, tornando-se relevante para o cenário da saúde pública com o intuito de maximizar o investimento de recursos públicos (HOEHNER et al., 2013).

Em consonância, estudos que investigaram o nível de atividade física e percepção do indivíduo sobre o ambiente mostram a importância de programas de promoção de atividade física para a população. Estimula-se que os pesquisadores aprofundem o estudo das variáveis relacionadas à percepção de segurança e da estrutura física pública, tal como a iluminação e de lazer nos bairros. Dessa forma, as medidas de auto-relato de ambientes indicam uma relação positiva com a atividade física (DOWNS, 2016).

Sabemos que as indústrias de automobilismo, construção e entretenimento, contribuíram para o estilo de vida sedentário, assim, o planejamento das intervenções públicas na prevenção de inatividade física pode vir de estratégias na mudança deste contexto, como o incentivo ao transporte ativo, mudanças no ambiente construído e 
Carla Regina de S. Teixeira, Maria Lúcia Zanetti,

Camila de Moraes, Plínio Tadeu Istilli,

Gabriel Guidorizzi Zanetti, Tânia Alves C. Becker e

Jennifer Vieira P. Marques

oportunidades de entretenimento voltados à prática de atividade física (PARRA et al., 2016).

Um estudo de revisão sistemática sobre as estratégias utilizadas no tratamento de DM apontou que a prática de atividade física e a alimentação saudável, como tratamento não farmacológico é de baixo custo e tem implicação positiva na qualidade de vida (BELON e NYKIFORUK, 2013). No entanto, outro estudo mostrou que 56\% das pessoas com diabetes mellitus tipo 2 (DM2) não realizam qualquer tipo de atividade física no período de lazer (GONELA, 2010). Dessa forma, há lacunas na literatura sobre a percepção do ambiente para a prática de atividade física de pessoas com DM2, o que pode contribuir para a busca de evidências no planejamento e efetividade das ações em saúde pública.

Diante do exposto, este estudo teve como objetivo analisar a percepção do ambiente para a prática de atividade física de lazer (AFL) e caminhada como forma de deslocamento (CFD) de pessoas com DM2.

\section{Métodos}

Estudo quantitativo transversal realizado em um Centro de Saúde Escola no interior paulista. A população-base foi constituída por 98 pessoas com DM2 que participaram do estudo de Becker (2014). Para este estudo foram estabelecidos os seguintes critérios: registro de diagnóstico de DM2 do prontuário de saúde, idade entre 18 e 90 anos, ambos os sexos e ter respondido a totalidade dos questionários. Foram excluídos 12 sujeitos que não completaram as etapas da pesquisa citadas anteriormente, permanecendo 86 sujeitos no estudo. 
Carla Regina de S. Teixeira, Maria Lúcia Zanetti,

Camila de Moraes, Plínio Tadeu Istilli,

Gabriel Guidorizzi Zanetti, Tânia Alves C. Becker e

Jennifer Vieira P. Marques

Para a coleta de dados foram utilizados três instrumentos: um questionário contendo as variáveis sociodemográficas (sexo, idade, renda, escolaridade, estado civil, religião e ocupação), o Questionário Internacional de Atividade Física (IPAQ) versão longa (MATSUDO et al., 2001), a Escala de Percepção do Ambiente adaptada (FLORINDO et al., 2012).

O IPAQ na versão longa é um questionário que permite a avaliação do nível de atividade física. É composto por 27 questões relacionadas ao nível de atividade física (MATSUDO et al., 2001). Para este estudo foram eleitos os domínios atividade física de lazer (AFL) e a caminhada como forma de deslocamento (CFD).

Utilizou-se a classificação de atividade física recomendada para pessoas com DM2 que consiste em pelo menos 150 minutos por semana de atividade física moderada ou 75 minutos de atividade física vigorosa, ou uma combinação de ambos (DIRETRIZES DA SOCIEDADE BRASILEIRA DE DIABETES, 2016). Atividade física moderada é considerada quando o consumo de $\mathrm{VO} 2_{\text {máx }}$ (consumo máximo de oxigênio) é de 40-60\%, ou quando há facilidade de falar durante a realização da atividade física. A atividade física vigorosa refere-se ao consumo de $\mathrm{VO} 2_{\text {máx }}>60 \%$, ou quando há dificuldade de se comunicar durante a atividade (DIRETRIZES DA SOCIEDADE BRASILEIRA DE DIABETES, 2016).

Neste estudo, utilizamos a escala validada por Florindo et al. (2012) composta por questões embasadas na escala Neighborhood Environmental Walkability Scale (NEWS - versão brasileira) (MALAVASI et al., 2007) e numa escala de apoio social para a prática de atividade física (REIS; REIS e HALLAL 2011), que é recomendada para estudos com o objetivo de avaliar a percepção do ambiente para a prática de atividade física em adultos e idosos. Em relação aos coeficientes de correlação para 
Carla Regina de S. Teixeira, Maria Lúcia Zanetti,

Camila de Moraes, Plínio Tadeu Istilli,

Gabriel Guidorizzi Zanetti, Tânia Alves C. Becker e

Jennifer Vieira P. Marques

reprodutibilidade, variaram de $\mathrm{r}=0,51$ para questão de acesso a agências bancárias a $\mathrm{r}=0,89$ para o acesso a supermercados, e para os escores de $\mathrm{r}=0,72$ para o escore de poluição geral a até $\mathrm{r}=0,94$ para o escore de facilidades/conveniências, apresentando resultados satisfatórios de reprodutibilidade para a amostra de idosos e de validade para os adultos, principalmente, para a percepção de acessibilidade a estruturas de conveniências/facilidades, percepção de segurança geral e de apoio social para a prática de atividade física.

A versão final é composta de 38 questões e pode ser utilizada para conhecer a relação entre ambiente e atividade física na comunidade. As questões são relacionadas à percepção do indivíduo em relação às estruturas físicas e ambientais próximas de sua residência, bem como o suporte social para a prática de atividades físicas e aspectos climáticos. $\mathrm{O}$ instrumento inclui questões sobre proximidade de lojas e comércio no geral, densidade residencial, percepção do acesso a esses locais, características das ruas, facilidades para caminhar e andar de bicicleta, arredores do bairro e segurança em relação ao tráfego e aos crimes (FLORINDO et al., 2012).

A coleta de dados sociodemográficos, nível de atividade física e percepção do ambiente foi realizada no período de julho a setembro de 2013, no Centro de Saúde Escola, pelos pesquisadores, em ambiente privativo, com duração média de trinta minutos por meio de entrevista.

Os dados coletados foram, inicialmente, organizados e digitados e submetidos à dupla validação no programa Microsoft Excel, versão XP (Microsoft Co, USA). Os resultados foram descritos por meio de média e desvio padrão (DP) para as variáveis quantitativas; e se qualitativas por meio de proporções. Os dados foram apresentados por meio de tabelas e de medidas descritivas. 
Carla Regina de S. Teixeira, Maria Lúcia Zanetti,

Camila de Moraes, Plínio Tadeu Istilli,

Gabriel Guidorizzi Zanetti, Tânia Alves C. Becker e

Jennifer Vieira P. Marques

No presente estudo foram realizados dois modelos de análise de regressão logística, em um deles a variável dependente do estudo foi a prática de AFL e a prática de CFD. Em ambas as variáveis as categorias referem-se à prática de pelo menos 150 minutos por semana.

Para realizar a análise de regressão foi utilizado inicialmente o procedimento de seleção denominado 'stepwise', pelo método 'forward'. A estatística de Wald foi utilizada para a seleção das variáveis (valores com $\mathrm{p}<0.20$ entravam no modelo). Esse processo foi realizado para o conjunto de todas as variáveis independentes de interesse. Deste grupo foi obtido o modelo 1, e foram inseridas as variáveis sociodemográficas, como sexo (masculino/feminino), idade (em anos) e estudos em anos (0 a 4 anos, 5 a 8 anos e 9 ou mais anos) para verificar possíveis efeitos de confundimento com relação as variáveis do modelo 1. Em todos os testes foi adotado o nível de significância de 5\% $($ alfa $=0.05)$. Para o ajuste dos modelos foi utilizado o programa IBM SPSS versão 22.

A pesquisa foi aprovada pelo Comitê de Ética e Pesquisa da Escola de Enfermagem de Ribeirão Preto - SP, sob Protocolo de $n^{0} 324.098$ e base de dados de pesquisas clínicas em seres humanos denominada ClinicalTrials.gov, sob Protocolo de $\mathrm{n}^{\mathrm{o}}$ NCT01972412 (BRASIL, 2012).

\section{Resultados}

Das 86 pessoas com DM2, a maioria tinha idade superior a 60 anos, eram mulheres e casados, católicos, aposentados e com renda de um a três salários mínimos. A média de idade foi de 63,61 ( $\mathrm{dp}=9,56$ anos). A escolaridade média foi de 6,45 (dp=4,15 anos), indicando baixo nível socioeconômico (TABELA 1). 
Carla Regina de S. Teixeira, Maria Lúcia Zanetti,

Camila de Moraes, Plínio Tadeu Istilli,

Gabriel Guidorizzi Zanetti, Tânia Alves C. Becker e

Jennifer Vieira P. Marques

Tabela 1: Caracterização das variáveis sociodemográficas dos sujeitos, Ribeirão Preto SP, 2013.

\begin{tabular}{lcr}
\hline Variáveis Sociodemográficas / Média (DP*) & n(86) & \% \\
\hline Idade (anos) / 63,61 (9,56) & 49 & 56,98 \\
$<65$ anos & 37 & 43,02 \\
$\geq 65$ anos & & \\
& & 72,09 \\
Sexo & 62 & 27,91 \\
Feminino & 24 & \\
Masculino & & 2,33 \\
& & 72,09 \\
Estado civil & 2 & 17,44 \\
Solteiro & 62 & 8,14 \\
Casado & 15 & 7
\end{tabular}

Escolaridade (anos) / 6,45 $(4,15)$

1 a 4

5 a 8

$\geq 9$

$23 \quad 26,74$

Sem escolaridade

$3 \quad 3,49$

\section{Ocupação/Profissão}

Carteira assinada

Emprego informal

9,30

Aposentado

55,81

Do lar

22,09

Outros

$\begin{array}{ll}48 & 55,81 \\ 19 & 22,09\end{array}$

$6 \quad 6,98$

Renda familiar (R\$)**/ $971,41(857,54)$

1 a 3 salário

4 a 9 salários

$30 \quad 34,88$

mais de 10 salários

$4 \quad 4,65$

\section{Religião}

Católica

Evangélica

Espírita

Sem religião

Outras

*DP: desvio-padrão.

** Mediana (Intervalo) da Renda $=678$ / Salário mínimo vigente em $2013=\mathrm{R} \$ 678,00$. 
Carla Regina de S. Teixeira, Maria Lúcia Zanetti,

Camila de Moraes, Plínio Tadeu Istilli,

Gabriel Guidorizzi Zanetti, Tânia Alves C. Becker e

Jennifer Vieira P. Marques

Quanto a classificação da prática de atividade física de pelo menos 150 minutos de AFL e de 150 minutos de CFD, 58 (67,44\%) pacientes com DM2 referiram que não realizavam.

Em relação à percepção do ambiente os principais locais percebidos pelos pacientes com DM2 como perto ou que levariam tempo igual ou menor que 10 minutos para chegar caminhando a partir de sua residência foram à praça $(45,51 \%)$, o local especifico para caminhar $(47,70 \%)$, o ponto de ônibus $(94,19 \%)$, a farmácia $(56,98 \%)$, a igreja/templo religioso $(54,65 \%)$, a padaria $(87,21 \%)$, o bar $(82,60 \%)$ e o mercadinho $(65,12 \%)$. Os principais locais identificados como longe, ou que levariam tempo superior a 10 minutos de caminhada para chegar, foram o posto de saúde $(54,65 \%)$ e o supermercado $(45,30 \%)$.

Os locais adequados referidos para a prática de atividade física na percepção da maioria dos pacientes com DM2 foram o parque $(67,44 \%)$, o clube $(70,93 \%)$, a quadra de esportes (52,33\%), o campo de futebol (69,77\%) e a academia de ginástica/musculação (43,02\%). Por outro lado, aproximadamente $50 \%$ dos pacientes com DM2 referiram que existe uma praça $(46,51 \%)$ ou um local especifico para caminhar (45,35\%) há 10 minutos ou menos de sua residência.

Em relação à percepção de estrutura física e ambiental para a prática de atividade física, a maioria referiu que o trânsito dificulta a prática de caminhada ou o uso de bicicleta. Também foram referidas como dificuldades a ausência de faixa para travessia de pedestres, a falta de segurança durante a noite para caminhar, andar de bicicleta ou praticar esportes, carência de convites de amigos, vizinhos, ou parentes para fazer companhia para caminhar, andar de bicicleta ou praticar esporte e a escassez de eventos esportivos e/ou caminhadas no bairro. 
Carla Regina de S. Teixeira, Maria Lúcia Zanetti,

Camila de Moraes, Plínio Tadeu Istilli,

Gabriel Guidorizzi Zanetti, Tânia Alves C. Becker e

Jennifer Vieira P. Marques

Quanto à percepção do ambiente no que se refere à qualidade das calçadas para caminhar e das áreas verdes, os pacientes com DM2 as classificaram como boas e regulares. No que se refere aos escores do ambiente, a maioria dos sujeitos classificou os itens de segurança no trânsito, segurança geral, apoio social e poluição, como ruins e regulares (TABELA 2).

Tabela 2: Escores da percepção do ambiente, segundo A-NEWS, Ribeirão Preto, 2013.

\begin{tabular}{lcccccccc}
\hline $\begin{array}{l}\text { Percepção } \\
\text { Ambiente }- \\
\text { A-NEWS }\end{array}$ & $\begin{array}{c}\text { do } \\
\text { segurança no } \\
\text { trânsito }\end{array}$ & \multicolumn{2}{c}{$\begin{array}{c}\text { Escore de } \\
\text { segurança geral }\end{array}$} & $\begin{array}{c}\text { Escore de } \\
\text { Apoio Social }\end{array}$ & \multicolumn{2}{c}{ Escore de } & \multicolumn{2}{c}{ Escore de } \\
\hline \multirow{2}{*}{ Ruim } & $\mathbf{n}$ & $\mathbf{\%}$ & $\mathbf{n}$ & $\mathbf{\%}$ & $\mathbf{n}$ & $\mathbf{\%}$ & $\mathbf{n}$ & $\mathbf{\%}$ \\
\cline { 2 - 9 } Regular & 18 & 20,90 & 15 & 17,40 & 37 & 43,00 & 35 & 40,70 \\
Bom & 41 & 47,70 & 41 & 47,70 & 28 & 32,60 & 28 & 32,60 \\
Ótimo & 18 & 20,90 & 28 & 32,60 & 18 & 20,90 & 17 & 19,80 \\
& 9 & 10,50 & 2 & 2,30 & 3 & 3,50 & 6 & 7,00 \\
\hline
\end{tabular}

Para variável dependente da prática de AFL, as variáveis relacionadas à existência de quadras de esportes, supermercado, convite de vizinhos e/ou amigos para a prática e clima, apresentaram significância estatística. As pessoas que perceberam quadras de esportes próximas à sua residência têm 15,51 vezes mais chance de praticarem AFL do que as pessoas que disseram não perceberam. As pessoas que perceberam o supermercado próximo de sua residência têm 9,82 vezes mais chance de praticarem AFL do que as pessoas que não perceberam. Já para os que receberam convite de vizinho eles têm 25,26 vezes mais chance de praticarem AFL do que as pessoas que não receberam. Sobre o clima, a chance de praticarem AFL dentre os que não relatam dificuldade é 0,13 vezes maior do que os que relataram dificuldade.

Agora, considerando-se a presença das variáveis sociodemográficas tem-se que as pessoas perceberam quadras de esportes próximas à sua residência têm 17,03 vezes 
Carla Regina de S. Teixeira, Maria Lúcia Zanetti,

Camila de Moraes, Plínio Tadeu Istilli,

Gabriel Guidorizzi Zanetti, Tânia Alves C. Becker e

Jennifer Vieira P. Marques

mais chance de praticarem AFL do que as pessoas que não perceberam quadras. As pessoas que perceberam supermercado próximo à sua residência, 11,44 vezes mais chance de praticarem AFL do que as pessoas que não perceberam. E as pessoas que receberam convites de vizinhos e/ou amigos para prática, têm 32,25 vezes mais chance de praticarem AFL do que as pessoas que não receberam. Sobre a dificuldade do clima tem-se que a chance de praticarem AFL dentre os que não relatam dificuldade é 0,10 vezes maior do que os que relataram dificuldade (TABELA 3).

Tabela 3: Modelo final entre percepção do ambiente e a prática de atividade física de lazer. Ribeirão Preto, SP, 2013.

\begin{tabular}{|c|c|c|c|}
\hline \multirow[b]{2}{*}{ Variável } & \multicolumn{3}{|c|}{ Modelo Final $^{\mathrm{a}}$} \\
\hline & $\mathrm{RP}$ & IC95\% & $\mathrm{p}$ \\
\hline \multicolumn{4}{|l|}{ Atividade física de lazer } \\
\hline Existência de quadra de esportes & & & 0,005 \\
\hline Não tem & & 1 & \\
\hline Tem a mais de 10 minutos caminhando da residência & 0,49 & 0,$06 ; 4,05$ & \\
\hline Tem a menos de 10 minutos caminhando da residência & 17,03 & 2,$38 ; 121,66$ & \\
\hline Existência de Supermercado & & & 0,037 \\
\hline Não tem & & 1 & \\
\hline Tem a mais de 10 minutos caminhando da residência & 0,45 & 0,$06 ; 3,57$ & \\
\hline Tem a menos de 10 minutos caminhando da residência & 11,44 & 1,$16 ; 112,57$ & \\
\hline Convite de amigos e vizinhos para a prática & & & 0,001 \\
\hline Não recebe & & 1 & \\
\hline Recebe & 32,25 & 4,$02 ; 258,65$ & \\
\hline Clima dificulta a prática & & & 0,028 \\
\hline Sim & & 1 & \\
\hline Não & 0,10 & 0,$014 ; 0,78$ & \\
\hline
\end{tabular}

${ }^{\text {a }}$ Modelo ajustado por sexo, idade, escolaridade

Com relação à variável dependente da prática de CFD tem-se que as variáveis que identificam existência de feira e ruas planas próximas à residência apresentaram significância estatística. Pessoas que perceberam a feira longe de sua residência têm 
Carla Regina de S. Teixeira, Maria Lúcia Zanetti,

Camila de Moraes, Plínio Tadeu Istilli,

Gabriel Guidorizzi Zanetti, Tânia Alves C. Becker e

Jennifer Vieira P. Marques

6,46 vezes mais chance de praticarem CFD do que as pessoas que não perceberam. As pessoas que perceberam que próximo à sua residência existem ruas planas têm 5,37 vezes mais chance de praticar CFD do que as pessoas que não perceberam.

Ao acrescentar às variáveis sociodemográficas, as variáveis da percepção continuam estatisticamente significantes. Na presença delas tem-se que as pessoas que perceberam a feira longe de sua residência têm 8,24 vezes mais chance de praticarem CFD do que as pessoas que não perceberam enquanto que as pessoas que perceberam ruas planas próximas à sua residência têm 5,86 vezes mais chance de praticar CFD do que as pessoas que não perceberam (TABELA 4).

Tabela 4: Modelo final ajustado entre percepção do ambiente e a prática caminhada como forma de deslocamento. Ribeirão Preto, SP, 2013.

\begin{tabular}{|c|c|c|c|}
\hline \multirow[b]{2}{*}{ Variável } & \multicolumn{3}{|c|}{ Modelo Final $^{\mathrm{a}}$} \\
\hline & $\mathrm{RP}$ & IC95\% & $\mathrm{p}$ \\
\hline \multicolumn{4}{|l|}{ Caminhada como forma de deslocamento } \\
\hline Existência de feira & & & 0,020 \\
\hline Não tem & & 1 & \\
\hline Tem a mais de 10 minutos caminhando da residência & 8,28 & 1,$57 ; 43,77$ & \\
\hline Tem a menos de 10 minutos caminhando da residência & 0,63 & 0,$10 ; 4,01$ & \\
\hline Ruas planas próximas a residência & & & 0,022 \\
\hline Não & & 1 & \\
\hline Sim & 5,86 & 1,$28 ; 26,73$ & \\
\hline
\end{tabular}

${ }^{\text {a }}$ Modelo ajustado por sexo, idade, escolaridade

\section{Discussão}

Ao analisar as variáveis sociodemográficas constata-se que os nossos achados corroboram aos da literatura. Estudos apontam que a aposentadoria, a idade, a baixa renda e escolaridade podem influenciar o nível de atividade física e percepção do ambiente dos indivíduos. Nessa direção, a estrutura física e ambiental dos espaços para 
Carla Regina de S. Teixeira, Maria Lúcia Zanetti,

Camila de Moraes, Plínio Tadeu Istilli,

Gabriel Guidorizzi Zanetti, Tânia Alves C. Becker e

Jennifer Vieira P. Marques

a prática de AFL e de CFD a população em geral, e em particular, aos pacientes com DM2 tem sido considerada variáveis importantes para que pacientes idosos com DM2 e com baixo poder aquisitivo possam realizar a atividade física em locais com segurança e iluminação adequada nos bairros (FLORINDO et al., 2011; REIS et al., 2009; ROCHA et al., 2013; WHITING et al., 2011).

Reconhece-se que há evidências na literatura de que a prática regular de atividade física é um importante coadjuvante para a obtenção de um bom controle metabólico. No entanto, a adesão à prática recomendada é baixa, apontando que ainda é preciso aprofundar a investigação se a percepção do ambiente pelos indivíduos pode ampliar a compreensão deste fenômeno (ZANETTI et al., 2014). Ao considerar que a prevalência de atividade física como forma de deslocamento na população brasileira é baixa, há necessidade de buscar estratégias efetivas para o aumento dos níveis de atividade física geral e para melhoria da saúde (MADEIRA et al., 2013).

Ao analisar a qualidade da estrutura física e ambiental no ambiente próximas às residências constatou-se que estas foram classificadas como boa para a atividade física de lazer. Essa percepção pode estar relacionada ao estímulo que os pacientes com DM2 tem para desenvolverem as atividades, o apoio social recebido, os programas de saúde para o incremento da atividade física e criação de ambientes propícios que permite considerar as características típicas de estilo de vida fisicamente ativo ou inativo (SOFKOVÁ et al., 2013). No entanto, apesar da qualidade da estrutura física e ambiental encontrou-se que a maioria dos sujeitos não praticava $\mathrm{AFL}$ e ou $\mathrm{CFD}$, de acordo com as recomendações do tratamento das pessoas com DM.

Em relação ao ambiente, os locais considerados mais propícios para a prática de atividade física, foram percebidos como longe da residência dos sujeitos, e a maior 
Carla Regina de S. Teixeira, Maria Lúcia Zanetti,

Camila de Moraes, Plínio Tadeu Istilli,

Gabriel Guidorizzi Zanetti, Tânia Alves C. Becker e

Jennifer Vieira P. Marques

parte do ambiente percebido foi considerada boa ou regular. Em relação à percepção do ambiente e a prática de AFL, obteve-se que quando os participantes percebiam a existência de quadras de esportes e supermercado próximos a sua residência, estes eram mais ativos dos que os que não percebiam. E que os participantes que recebem convite de vizinhos e/ou amigo têm mais chance de praticarem do que as pessoas que não recebem, e o clima pode dificulta a prática. As pessoas que percebem a feira longe de sua residência mais chance de praticarem CFD do que as pessoas que não percebem. Assim como, as pessoas que percebem ruas planas próximas à sua residência têm mais chance de praticar do que as pessoas que não percebem.

Assim, devemos levar em consideração pessoas com algumas condições crônicas como o DM, como mostra um estudo que associou as características do ambiente e atividade física em idosos com tipos específicos de doenças crônicas, e concluiu que aumentar o nível de atividade física em adultos mais velhos que têm níveis mais baixos de funcionalidade física devido às condições crônicas podem exigir características específicas da vizinhança (BARNETT, et al., 2016).

\section{Considerações Finais}

A percepção do ambiente tem relação com a prática de atividade física no lazer e na caminhada como meio de deslocamento. A existência de locais acessíveis mais próximos das residências parece propiciar a prática de atividade física de lazer, assim como o ambiente social é de suma importância para o estímulo à prática. O ambiente com ruas mais planas próximos das residências parece que facilita a prática de caminhada como forma de deslocamento. A utilização de espaços públicos adequados propicia a prática de AFL e CFD, como parte do tratamento da doença, bem como, o 
Carla Regina de S. Teixeira, Maria Lúcia Zanetti,

Camila de Moraes, Plínio Tadeu Istilli,

Gabriel Guidorizzi Zanetti, Tânia Alves C. Becker e

Jennifer Vieira P. Marques

apoio social entre amigos e familiares e o suporte contínuo para a manutenção das atividades. Concluímos que o ambiente físico e social quando percebido de forma positiva, serve como um facilitador para a prática de atividade física recomendada para a população, e encontrar dados sobre a percepção do ambiente de determinada população, como quem possui DM2, visto que podem apresentar condições físicas, psicológicas e motivacionais diferentes de pessoas sem doenças crônicas, pode contribuir para melhor adequação nas ações de promoção de atividade física da comunidade. Por fim, o estímulo, por parte dos profissionais de saúde, a utilização do ambiente físico e social para o benefício da saúde da pessoa com DM2 caracterizou-se como uma intervenção importante no tocante ao gerenciamento adequado do tratamento desta condição crônica de saúde.

\section{Agradecimentos}

Ao CNPq, pela concessão do fomento para o desenvolvimento da pesquisa, proveniente do Edital Universal $\mathrm{n}^{\mathrm{o}}$ 42/2010, processo $\mathrm{n}^{\mathrm{o}}$ 563598/2010-7-MCT/CTSaúde/MS/SCTIE/DECIT. A CAPES, pela concessão da bolsa de estudo do Mestrado, pela Escola de Enfermagem de Ribeirão Preto / USP. Aos membros do Grupo de Pesquisa em Enfermagem e Diabetes Mellitus da EERP/USP. Agradecimento especial ao Prof. Alex Florindo pelas sugestões e esclarecimentos para enriquecimento do trabalho.

\section{REFERÊNCIAS}

BARNETT, et al. A. et al. Associations between the neighbourhood environment characteristics and physical activity in older adults with specific types of chronic conditions: the ALECS cross-sectional study. International Journal of Behavioral Nutrition and Physical Activity, London, v. 13, n. 1, p. 1, 2016. 
Carla Regina de S. Teixeira, Maria Lúcia Zanetti,

Camila de Moraes, Plínio Tadeu Istilli,

Gabriel Guidorizzi Zanetti, Tânia Alves C. Becker e

Jennifer Vieira P. Marques

BECKER, T. A. C. O uso do suporte telefônico no controle metabólico de pessoas com diabetes mellitus no Distrito Oeste de Saúde do município de Ribeirão Preto SP. 2014. Tese (Doutorado em Enfermagem Fundamental) - Escola de Enfermagem de Ribeirão Preto, Universidade de São Paulo, Ribeirão Preto, 2014. Disponível em: http://www.teses.usp.br/teses/disponiveis/22/22132/tde-09022015-195419/ . Acesso em: 31 mar. 2015.

BELON, A.; NYKIFORUK, C. Possibilities and challenges for physical and social environment research in Brazil: a systematic literature review on health behaviors. Cadernos de Saúde Pública, Rio de Janeiro, v. 29, n. 10, p. 1955-1973, Oct. 2013.

BRASIL. Conselho Nacional de Saúde. Resolução n 466, de 12 de dezembro de 2012. Aprova as diretrizes e normas regulamentadoras de pesquisas envolvendo seres humanos. Resolução Brasília: CNS, 2012. Disponível em: <http://conselho.saude.gov.br

COLBERG, S.R.; HERNANDEZ, M.J.; SHAHZAD, F. Blood glucose responses to type, intensity, duration, and timing of exercise. Diabetes Care, v. 36, n. 10, p. 177, 2013.

DIRETRIZES DA SOCIEDADE BRASILEIRA DE DIABETES (2015-2016) / Adolfo Milech...[et al.]; organização José Egidio Paulo de Oliveira, Sérgio Vencio - São Paulo: A.C. Farmacêutica, 2016.

DOWNS, A. Physically Active Adults: An Analysis of the Key Variables That Keep Them Moving. American Journal of Health Education, Chicago, v. 47, n. 5, p. 299308, 2016.

FERMINO, R.; REIS, R. Variáveis individuais, ambientais e sociais associadas com o uso de espaços públicos abertos para a prática de atividade física: uma revisão sistemática. Revista Brasileira de Atividade Física \& Saúde, v. 18, n. 5, p. 523, 2014.

FLORINDO, A. A. et al. Perception of the environment and practice of physical activity by adults in a low socioeconomic area. Revista de Saúde Pública, São Paulo, v. 45, n. 2, p. 302-310, apr. 2011. Disponível em: http://www.ncbi.nlm.nih.gov/pubmed/21412570 . Acesso em: 27 jan. 2016.

FLORINDO, A. A. et al. Validação de uma escala de percepção do ambiente para a prática de atividade física em adultos de uma região de baixo nível socioeconômico. Revista Brasileira de Cineantropometria e Desempenho Humano, Florianópolis, v. 14, n. 6, p. 647-659, dez. 2012. Disponível em:http://www.scielo.br/scielo.php?script=sci_arttext\&pid=S198000372012000600004\&lng=en\&nrm=iso . Acesso em: 27 jan. 2016.

GONELA, J. T. Nível de atividade física em pessoas com diabetes mellitus tipo 2. 2010. Dissertação (Mestrado em Enfermagem Fundamental) - Escola de Enfermagem de Ribeirão Preto, Universidade de São Paulo, Ribeirão Preto, 2010. Disponível em: http://www.teses.usp.br/teses/disponiveis/22/22132/tde-16112010-100753/ . Acesso em: 24 jan., 2014. 
Carla Regina de S. Teixeira, Maria Lúcia Zanetti,

Camila de Moraes, Plínio Tadeu Istilli,

Gabriel Guidorizzi Zanetti, Tânia Alves C. Becker e

Jennifer Vieira P. Marques

HOEHNER, C.M. et al. Physical activity interventions in Latin America: expanding and classifying the evidence. American Journal of Preventive Medicine, v. 44, n. 3, p. e31-e40, 2013.

MADEIRA, M. C. et al. Atividade física no deslocamento em adultos e idosos do Brasil: prevalências e fatores associados. Caderno de Saúde Pública, v. 29, n. 1, p. 165-174, 2013.

MALAVASI, L. et al. Escala de mobilidade ativa no ambiente comunitário - news Brasil: retradução e reprodutibilidade. Revista Brasileira de Cineantropometria e Desempenho Humano, Florianópolis, v. 9, n. 4, p. 339-350, 2007.

MALTA, D. C. et al. Prevalência de fatores de risco e proteção para doenças crônicas não transmissíveis em adultos residentes em capitais brasileiras, 2013. Epidemiol. Serv. Saúde, Brasília, v. 24, n. 3, p. 373-387, Sept. 2015.

MATSUDO, S. et al. Questionário internacional de atividade física (IPAQ): estudo de validade e reprodutibilidade no Brasil. Revista Brasileira de Atividade Física \& Saúde, Londrina, v. 6, n. 2, p. 05-18, 2001.

PARRA, D. C. et al. Automobile, construction and entertainment business sector influences on sedentary lifestyles. Health promotion international, p. daw073, 2016.

REIS, R. S. et al. Association between physical activity in parks and perceived environment: a study with adolescents. Journal of Physical Activity \& Health, Champaign, v. 6, n. 4, p. 503, 2009.

REIS, M. S. D.; REIS, R. S.; HALLAL, P. C. Validade e fidedignidade de uma escala de avaliação do apoio social para a atividade física. Revista de Saúde Pública, São Paulo, v. 45, n. 2, p. 294-301, 2011.

ROCHA, S. V. et al. Fatores associados à atividade física insuficiente no lazer entre idosos. Revista Brasileira de Medicina do Esporte, São Paulo, v. 19, n. 3, p. 191-195, 2013.

SALLIS, J. F. et al. Progress in physical activity over the olympic quadrennium. Lancet, v. 6736 n. 16, p. 1-12, 2016.

SOFKOVÁ, T. et al. The level of neighborhood walkability in a place of residence and its effect on body composition in obese and overweight women. Central European Journal of Public Health, Prague, v. 21, n. 4, p. 184-189, 2013.

WORLD HEALTH ORGANIZATION (WHO). Noncommunicable diseases country profiles 2014. Geneva, 2014. 
Carla Regina de S. Teixeira, Maria Lúcia Zanetti,

Camila de Moraes, Plínio Tadeu Istilli,

Gabriel Guidorizzi Zanetti, Tânia Alves C. Becker e

Jennifer Vieira P. Marques

WHITING, D. R. et al. IDF Diabetes Atlas: global estimates of the prevalence of diabetes for 2011 and 2030. Diabetes Research and Clinical Practice, Amsterdam, v. 94, n. 3, p. 311-321, 2011. Disponível em: http://www.sciencedirect.com/science/article/ pii/S0168822711005912 . Acesso em: 20 jan. 2014.

ZANETTI, G. G. et al. Investigating telephone support as a strategy to increase the physical activity levels of people with diabetes. Journal of Diabetes Nursing, London, v. 18, n. 1, p. 32-36, 2014.

\section{Endereço dos Autores:}

Paula Parisi Hodniki,

Escola de Enfermagem de Ribeirão Preto

Universidade de São Paulo (USP)

Avenida dos Bandeirantes número 3900,

Campus Universitário - Bairro Monte Alegre

Ribeirão Preto - SP - 14.040-902

Endereço Eletrônico: paulaph@hotmail.com

Carla Regina de Souza Teixeira

Escola de Enfermagem de Ribeirão Preto

Universidade de São Paulo (USP)

Avenida dos Bandeirantes número 3900,

Campus Universitário - Bairro Monte Alegre

Ribeirão Preto - SP - 14.040-902

Endereço Eletrônico: carlarst@eerp.usp.br

Maria Lúcia Zanetti

Escola de Enfermagem de Ribeirão Preto

Universidade de São Paulo (USP)

Avenida dos Bandeirantes número 3900,

Campus Universitário - Bairro Monte Alegre

Ribeirão Preto - SP - 14.040-902

Endereço Eletrônico: zanetti@eerp.usp.br

Camila de Moraes

Escola de Educação Física e Esporte de Ribeirão Preto

Universidade de São Paulo

Avenida Bandeirantes, 3900 - Vila Monte Alegre

Ribeirão Preto - SP - 14.040-030

Endereço Eletrônico: camimoraes@usp.br 
Carla Regina de S. Teixeira, Maria Lúcia Zanetti,

Camila de Moraes, Plínio Tadeu Istilli,

Gabriel Guidorizzi Zanetti, Tânia Alves C. Becker e

Jennifer Vieira P. Marques

Plínio Tadeu Istilli

Escola de Enfermagem de Ribeirão Preto

Universidade de São Paulo (USP)

Avenida dos Bandeirantes número 3900,

Campus Universitário - Bairro Monte Alegre

Ribeirão Preto - SP - 14.040-902

Endereço Eletrônico:istilli@hotmail.com

Gabriel Guidorizzi Zanetti

Escola de Enfermagem de Ribeirão Preto

Universidade de São Paulo (USP)

Avenida dos Bandeirantes número 3900,

Campus Universitário - Bairro Monte Alegre

Ribeirão Preto - SP - 14.040-902

Endereço Eletrônico: gabriel.guidorizzi@gmail.com

Tânia Alves Canata Becker

Escola de Enfermagem de Ribeirão Preto

Universidade de São Paulo (USP)

Avenida dos Bandeirantes número 3900,

Campus Universitário - Bairro Monte Alegre

Ribeirão Preto - SP - 14.040-902

Endereço Eletrônico: taniacanata@gmail.com

Jennifer Vieira Paschoalin Marques

Escola de Enfermagem de Ribeirão Preto

Universidade de São Paulo (USP)

Avenida dos Bandeirantes número 3900,

Campus Universitário - Bairro Monte Alegre

Ribeirão Preto - SP - 14.040-902

Endereço Eletrônico: jevieirapaschoalin@hotmail.com 